$\int \mathbb{M}$\begin{tabular}{lr} 
Journal of Applied Mathematics and Computational Mechanics 2020, 19(3), 111-121 \\
$\begin{array}{ll}\text { www.amcm.pcz.pl } & \text { p-ISSN 2299-9965 } \\
\text { DOI: 10.17512/jamcm.2020.3.09 } & \text { e-ISSN 2353-0588 }\end{array}$ \\
\hline
\end{tabular}

\title{
ANALYSIS OF THE THREE-DIMENSIONAL THERMOELASTICITY PROBLEM WITH THE USE OF THE CONTINUOUS GALERKIN METHOD
}

\author{
Ewa Wegrzyn-Skrzypczak \\ Department of Mathematics, Czestochowa University of Technology \\ Czestochowa, Poland \\ ewa.skrzypczak@im.pcz.pl
}

Received: 6 July 2020; Accepted: 17 August 2020

\begin{abstract}
The paper focuses on the mathematical and numerical modelling of the thermoelasticity problem in the three-dimensional region. The governing equations of the mathematical model are a set of equilibrium equations. The numerical model uses continuous Galerkin formulation together with the Finite Element Method (FEM). Both models are discussed in detail. The final set of FEM equations is derived. The example of numerical calculations obtained with the use of an original computer program is presented. The main goal of presented paper is to develop the alternative model to the one based on the Discontinuous Galerkin Method (DGM).
\end{abstract}

MSC 2010: 35J25, 65M60

Keywords: thermoelasticity, Finite Element Method, continuous Galerkin method

\section{Introduction}

Elastic deformations can be induced by thermal and mechanical loads. The solution of the problem of thermoelasticity is to determine the displacements of the material points in the considered body caused by the forces or temperature field [1]. The coupling of these two actions can be described by the appropriate differential equations.

In many cases, which are important from the technical point of view, finding a solution of the differential equation describing specific physical phenomena in analytical form is difficult or even impossible to achieve. Therefore, parallel to the development of analytical methods for finding exact solutions, approximate methods were developed and improved. In these methods, the problem of searching for unknown functions (describing e.g. displacement, strain and stress field) is replaced by the problem of searching for a finite number of parameters that can be used to describe - with some approximation - the sought functions. In technical 
sciences, approximate methods are usually described from the application side as specialized computational procedures for specific problems, for example to obtain elastic deformation fields, temperature fields, electric and magnetic fields. There are many approximate methods used in the mechanics of a deformable body, e.g. the Ritz, Galerkin, Trefftz and collocation methods [2-5]. Nowadays, the methods that are easy to implement using programming languages are very popular. Those that are the most known are the Finite Difference Method (FDM), the Finite Element Method (FEM) and the Boundary Element Method (BEM) [5-7].

The main purpose of presented work is to create an original solver for three-dimensional thermoelasticity problem based on the continuous approach and the FEM which is efficient, robust and free to use and makes obtaining results with the use of different orders of approximation possible.

\section{Mathematical and numerical description}

Let's consider a three-dimensional region $\Omega$ (Fig. 1). On the surfaces $\Gamma_{1}, \Gamma_{2}$ of the region, the displacements $u_{1}(x, y, z)$ and $u_{2}(x, y, z)$ are given respectively. In the whole region $\Omega$, the temperature distribution $T(x, y, z)$ is also known.

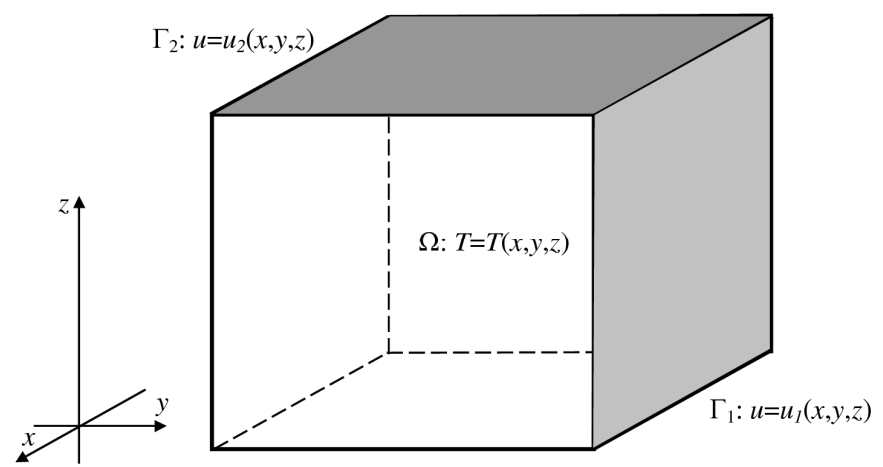

Fig. 1. Considered volume $\Omega$ with the temperature field $T(x, y, z)$ and displacements $u(x, y, z)$ on the boundaries $\Gamma_{1}, \Gamma_{2}$

The starting point of consideration is the system of three equilibrium equations:

$$
\begin{aligned}
& \frac{\partial \sigma_{x}}{\partial x}+\frac{\partial \tau_{x y}}{\partial y}+\frac{\partial \tau_{x z}}{\partial z}+F_{x}=0 \\
& \frac{\partial \tau_{x y}}{\partial x}+\frac{\partial \sigma_{y}}{\partial y}+\frac{\partial \tau_{y z}}{\partial z}+F_{y}=0 \\
& \frac{\partial \tau_{x z}}{\partial x}+\frac{\partial \tau_{y z}}{\partial y}+\frac{\partial \sigma_{z}}{\partial z}+F_{z}=0
\end{aligned}
$$


where $\sigma_{x}, \sigma_{y}, \sigma_{z}$ are normal stresses, $\tau_{x y}=\tau_{y x}, \tau_{x z}=\tau_{z x}, \tau_{y z}=\tau_{z y}$ are shear stresses, $F_{x}, F_{y}, F_{z}$ are components of the body forces vector. In the further consideration body forces $F_{x}, F_{y}, F_{z}$ are neglected.

To solve the system of equations (1) the criterion of the weighted residual $[7,8]$ is used. In this method, the differential equation is multiplied by a function called the weighting function $w$ and integrated over the considered domain $\Omega$, assuming that the integral equals zero. Using the criterion of the weighted residuals method for (1), one can obtain the following integral equations:

$$
\begin{aligned}
& \int_{\Omega} w\left(\frac{\partial \sigma_{x}}{\partial x}+\frac{\partial \tau_{x y}}{\partial y}+\frac{\partial \tau_{x z}}{\partial z}\right) d \Omega=0 \\
& \int_{\Omega} w\left(\frac{\partial \tau_{x y}}{\partial x}+\frac{\partial \sigma_{y}}{\partial y}+\frac{\partial \tau_{y z}}{\partial z}\right) d \Omega=0 \\
& \int_{\Omega} w\left(\frac{\partial \tau_{x z}}{\partial x}+\frac{\partial \tau_{y z}}{\partial y}+\frac{\partial \sigma_{z}}{\partial z}\right) d \Omega=0
\end{aligned}
$$

The weighting function $w$ can be any function of independent spatial coordinates. In order to write the weak form of equations (2), the order of each sub-integral equation must be lowered using Green's theorem:

$$
\begin{aligned}
& \int_{\Omega} w \frac{\partial \sigma_{x}}{\partial x} d \Omega=\oint_{\Gamma} w \sigma_{x} n_{x} d \Gamma-\int_{\Omega} \frac{\partial w}{\partial x} \sigma_{x} d \Omega \\
& \int_{\Omega} w \frac{\partial \tau_{x y}}{\partial y} d \Omega=\oint_{\Gamma} w \tau_{x y} n_{y} d \Gamma-\int_{\Omega} \frac{\partial w}{\partial y} \tau_{x y} d \Omega \\
& \int_{\Omega} w \frac{\partial \tau_{x z}}{\partial z} d \Omega=\oint_{\Gamma} w \tau_{x z} n_{z} d \Gamma-\int_{\Omega} \frac{\partial w}{\partial z} \tau_{x z} d \Omega
\end{aligned}
$$

where $\Gamma$ means the surface of $\Omega$.

The following relationships between stresses and displacements are used:

$$
\begin{gathered}
\sigma_{x}=f_{1} \frac{\partial u_{x}}{\partial x}+f_{2} \frac{\partial u_{y}}{\partial y}+f_{2} \frac{\partial u_{z}}{\partial z}-f_{4} \alpha \Delta T \\
\sigma_{y}=f_{2} \frac{\partial u_{x}}{\partial x}+f_{1} \frac{\partial u_{y}}{\partial y}+f_{2} \frac{\partial u_{z}}{\partial z}-f_{4} \alpha \Delta T \\
\sigma_{z}=f_{2} \frac{\partial u_{x}}{\partial x}+f_{2} \frac{\partial u_{y}}{\partial y}+f_{1} \frac{\partial u_{z}}{\partial z}-f_{4} \alpha \Delta T \\
\tau_{x y}=\tau_{y x}=f_{3} \frac{\partial u_{x}}{\partial y}+f_{3} \frac{\partial u_{y}}{\partial x} \\
\tau_{y z}=\tau_{z y}=f_{3} \frac{\partial u_{y}}{\partial z}+f_{3} \frac{\partial u_{z}}{\partial y} \\
\tau_{x z}=\tau_{z x}=f_{3} \frac{\partial u_{x}}{\partial z}+f_{3} \frac{\partial u_{z}}{\partial x}
\end{gathered}
$$


where $\Delta T$ is the difference between current and initial temperatures, $\alpha\left[\mathrm{K}^{-1}\right]$ is the linear coefficient of thermal expansion and $f_{1}, f_{2}, f_{3}, f_{4}$ are the coefficients listed below:

$$
\begin{gathered}
f_{1}=\frac{E(1-v)}{(1+v)(1-2 v)} \\
f_{2}=\frac{E v}{(1+v)(1-2 v)} \\
f_{3}=\frac{E}{2(1+v)} \\
f_{4}=\frac{E(1+v)}{(1+v)(1-2 v)}
\end{gathered}
$$

where $E\left[\mathrm{~N} / \mathrm{m}^{2}\right]$ is the Young's modulus and $v[-]$ represents Poisson's ratio.

The relationships (3)-(4) used in (2) make it possible to form the following integral equations depending on the displacements:

$$
\begin{gathered}
\int_{\Omega} \frac{\partial w}{\partial x} \sigma_{x} d \Omega=\int_{\Omega} \frac{\partial w}{\partial x}\left(f_{1} \frac{\partial u_{x}}{\partial x}+f_{2} \frac{\partial u_{y}}{\partial y}+f_{2} \frac{\partial u_{z}}{\partial z}-f_{4} \alpha \Delta T\right) d \Omega \\
\int_{\Omega} \frac{\partial w}{\partial y} \tau_{x y} d \Omega=\int_{\Omega} \frac{\partial w}{\partial y}\left(f_{3} \frac{\partial u_{x}}{\partial y}+f_{3} \frac{\partial u_{y}}{\partial x}\right) d \Omega \\
\int_{\Omega} \frac{\partial w}{\partial z} \tau_{x z} d \Omega=\int_{\Omega} \frac{\partial w}{\partial z}\left(f_{3} \frac{\partial u_{x}}{\partial z}+f_{3} \frac{\partial u_{z}}{\partial x}\right) d \Omega
\end{gathered}
$$

Using the equations (6) and the relationship $\sigma_{x} n_{x}+\tau_{x y} n_{y}+\tau_{x z} n_{z}=p_{x}$, where $p_{x}$ is the stress on the boundary in the direction of the $x$ axis, in relation to the first equation from the system (2), one can write:

$$
\begin{aligned}
& 0=\int_{\Omega} w\left(\frac{\partial \sigma_{x}}{\partial x}+\frac{\partial \tau_{x y}}{\partial y}+\frac{\partial \tau_{x z}}{\partial z}\right) d \Omega=\oint_{\Gamma} w p_{x} d \Gamma-\int_{\Omega} \frac{\partial w}{\partial x}\left(f_{1} \frac{\partial u_{x}}{\partial x}+f_{2} \frac{\partial u_{y}}{\partial y}+f_{2} \frac{\partial u_{z}}{\partial z}-f_{4} \alpha \Delta T\right) d \Omega+ \\
& -\int_{\Omega} \frac{\partial w}{\partial y}\left(f_{3} \frac{\partial u_{x}}{\partial y}+f_{3} \frac{\partial u_{y}}{\partial x}\right) d \Omega-\int_{\Omega} \frac{\partial w}{\partial z}\left(f_{3} \frac{\partial u_{x}}{\partial z}+f_{3} \frac{\partial u_{z}}{\partial x}\right) d \Omega
\end{aligned}
$$

Finally, the equation (7) can be written in the following form:

$$
\begin{aligned}
& \int_{\Omega}\left(f_{1} \frac{\partial w}{\partial x} \frac{\partial u_{x}}{\partial x}+f_{3} \frac{\partial w}{\partial y} \frac{\partial u_{x}}{\partial y}+f_{3} \frac{\partial w}{\partial z} \frac{\partial u_{x}}{\partial z}\right) d \Omega+\int_{\Omega}\left(f_{2} \frac{\partial w}{\partial x} \frac{\partial u_{y}}{\partial y}+f_{3} \frac{\partial w}{\partial y} \frac{\partial u_{y}}{\partial x}\right) d \Omega+ \\
& +\int_{\Omega}\left(f_{2} \frac{\partial w}{\partial x} \frac{\partial u_{z}}{\partial z}+f_{3} \frac{\partial w}{\partial z} \frac{\partial u_{z}}{\partial x}\right) d \Omega=\int_{\Omega} f_{4} \frac{\partial w}{\partial x} \alpha \Delta T d \Omega+\oint_{\Gamma} w p_{x} d \Gamma
\end{aligned}
$$


The other two equations from the system (2) are transformed analogously, i.e.:

$$
\begin{aligned}
& \int_{\Omega}\left(f_{2} \frac{\partial w}{\partial y} \frac{\partial u_{x}}{\partial x}+f_{3} \frac{\partial w}{\partial x} \frac{\partial u_{x}}{\partial y}\right) d \Omega+\int_{\Omega}\left(f_{3} \frac{\partial w}{\partial x} \frac{\partial u_{y}}{\partial x}+f_{1} \frac{\partial w}{\partial y} \frac{\partial u_{y}}{\partial y}+f_{3} \frac{\partial w}{\partial z} \frac{\partial u_{y}}{\partial z}\right) d \Omega+ \\
& +\int_{\Omega}\left(f_{2} \frac{\partial w}{\partial y} \frac{\partial u_{z}}{\partial z}+f_{3} \frac{\partial w}{\partial z} \frac{\partial u_{z}}{\partial y}\right) d \Omega=\int_{\Omega} f_{4} \frac{\partial w}{\partial y} \alpha T d \Omega+\oint_{\Gamma} w p_{y} d \Gamma \\
& \quad \int_{\Omega}\left(f_{2} \frac{\partial w}{\partial z} \frac{\partial u_{x}}{\partial x}+f_{3} \frac{\partial w}{\partial x} \frac{\partial u_{x}}{\partial z}\right) d \Omega+\int_{\Omega}\left(f_{2} \frac{\partial w}{\partial z} \frac{\partial u_{y}}{\partial y}+f_{3} \frac{\partial w}{\partial y} \frac{\partial u_{y}}{\partial z}\right) d \Omega+ \\
& +\int_{\Omega}\left(f_{3} \frac{\partial w}{\partial x} \frac{\partial u_{z}}{\partial x}+f_{3} \frac{\partial w}{\partial y} \frac{\partial u_{z}}{\partial y}+f_{1} \frac{\partial w}{\partial z} \frac{\partial u_{z}}{\partial z}\right) d \Omega=\int_{\Omega} f_{4} \frac{\partial w}{\partial z} \alpha T d \Omega+\oint_{\Gamma} w p_{z} d \Gamma
\end{aligned}
$$

Equations (8)-(10) are a weak form of equilibrium equations. Further considerations are made on the basis of the Bubnov-Galerkin formulation, which requires that weighting functions $w$ be assumed the same as the shape functions $\phi$ of the finite element.

Assume that the domain $\Omega$ are spatially discretized into a set of $N$ tetrahedrons:

$$
\Omega=\bigcup_{j=1}^{N} \Omega_{j}
$$

The normalized tetrahedron (Fig. 2) meets the following conditions in space $\mathbf{T}^{3}$ :

$$
\begin{aligned}
& -1 \leq r, s, t \leq 1 \\
& r+s+t \leq-1
\end{aligned}
$$

where $r, s, t$ are spatial coordinates.

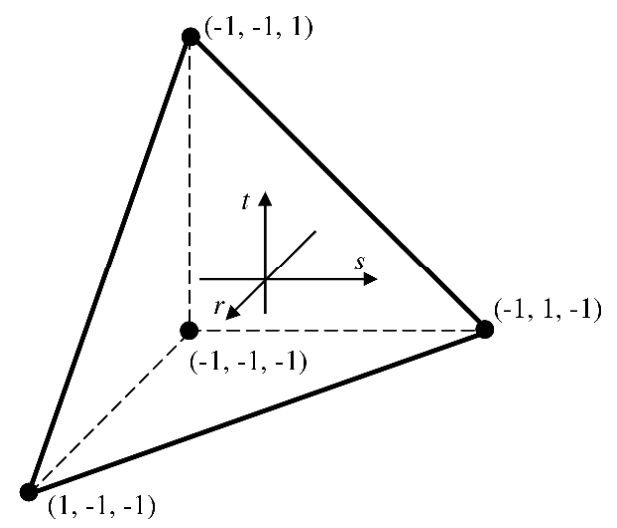

Fig. 2. Normalized tetrahedron described by coordinates $r, s, t$ 
The following spatial derivatives of the displacements and $\Delta T$ are approximated according to the formulas shown below:

$$
\begin{array}{ccc}
\frac{\partial u_{x}}{\partial x}=\sum_{n=0}^{M-1} \frac{\partial \varphi_{n}(r, s, t)}{\partial x} u_{n x}, & \frac{\partial u_{x}}{\partial y}=\sum_{n=0}^{M-1} \frac{\partial \varphi_{n}(r, s, t)}{\partial y} u_{n x}, & \frac{\partial u_{x}}{\partial z}=\sum_{n=0}^{M-1} \frac{\partial \varphi_{n}(r, s, t)}{\partial z} u_{n x} \\
\frac{\partial u_{y}}{\partial x}=\sum_{n=0}^{M-1} \frac{\partial \varphi_{n}(r, s, t)}{\partial x} u_{n y}, & \frac{\partial u_{y}}{\partial y}=\sum_{n=0}^{M-1} \frac{\partial \varphi_{n}(r, s, t)}{\partial y} u_{n y}, & \frac{\partial u_{y}}{\partial z}=\sum_{n=0}^{M-1} \frac{\partial \varphi_{n}(r, s, t)}{\partial z} u_{n y} \\
\frac{\partial u_{z}}{\partial x}=\sum_{n=0}^{M-1} \frac{\partial \varphi_{n}(r, s, t)}{\partial x} u_{n z}, & \frac{\partial u_{z}}{\partial y}=\sum_{n=0}^{M-1} \frac{\partial \varphi_{n}(r, s, t)}{\partial y} u_{n z}, & \frac{\partial u_{z}}{\partial z}=\sum_{n=0}^{M-1} \frac{\partial \varphi_{n}(r, s, t)}{\partial z} u_{n x} \\
\Delta T=\sum_{n=0}^{M-1} \varphi_{n}(r, s, t) \Delta T_{n} &
\end{array}
$$

where $M$ - number of shape functions for the tetrahedron (i.e. $M=4$ in the case of linear shape functions).

The relation $w=\phi$ together with (14) makes it possible to write equilibrium equations for a single finite element. For example, the subsequent integral parts of equation (8) are written as follows:

$$
\begin{gathered}
f_{1} \int_{\Omega^{(e)}} \frac{\partial w}{\partial x} \frac{\partial u_{x}}{\partial x} d \Omega^{(e)}=f_{1} \int_{\Omega^{(e)}} \frac{\partial \varphi_{n}}{\partial x} \frac{\partial \varphi_{m}}{\partial x} d \Omega^{(e)} u_{m x} \\
f_{3} \int_{\Omega^{(e)}} \frac{\partial w}{\partial y} \frac{\partial u_{x}}{\partial y} d \Omega^{(e)}=f_{3} \int_{\Omega^{(e)}} \frac{\partial \varphi_{n}}{\partial y} \frac{\partial \varphi_{m}}{\partial y} d \Omega^{(e)} u_{m x} \\
f_{3} \int_{\Omega^{(e)}} \frac{\partial w}{\partial z} \frac{\partial u_{x}}{\partial z} d \Omega^{(e)}=f_{3} \int_{\Omega^{(e)}} \frac{\partial \varphi_{n}}{\partial z} \frac{\partial \varphi_{m}}{\partial z} d \Omega^{(e)} u_{m x} \\
f_{2} \int_{\Omega^{(e)}} \frac{\partial w}{\partial x} \frac{\partial u_{y}}{\partial y} d \Omega^{(e)}=f_{2} \int_{\Omega^{(e)}} \frac{\partial \varphi_{n}}{\partial x} \frac{\partial \varphi_{m}}{\partial y} d \Omega^{(e)} u_{m y} \\
f_{3} \int_{\Omega^{(e)}} \frac{\partial w}{\partial y} \frac{\partial u_{y}}{\partial x} d \Omega^{(e)}=f_{3} \int_{\Omega^{(e)}} \frac{\partial \varphi_{n}}{\partial y} \frac{\partial \varphi_{m}}{\partial x} d \Omega^{(e)} u_{m y} \\
f_{2} \int_{\Omega^{(e)}} \frac{\partial w}{\partial x} \frac{\partial u_{z}}{\partial z} d \Omega^{(e)}=f_{2} \int_{\Omega^{(e)}} \frac{\partial \varphi_{n}}{\partial x} \frac{\partial \varphi_{m}}{\partial z} d \Omega^{(e)} u_{m z} \\
f_{3} \int_{\Omega^{(e)}} \frac{\partial w}{\partial z} \frac{\partial u_{z}}{\partial x} d \Omega^{(e)}=f_{3} \int_{\Omega^{(e)}} \frac{\partial \varphi_{n}}{\partial z} \frac{\partial \varphi_{m}}{\partial x} d \Omega^{(e)} u_{m z} \\
f_{4} \alpha \int_{\Omega^{(e)}} \frac{\partial w}{\partial x} \Delta T d \Omega^{(e)}=f_{4} \alpha \int_{\Omega^{(e)}} \frac{\partial \varphi_{n}}{\partial x} \varphi_{m} d \Omega^{(e)} \Delta T_{m}
\end{gathered}
$$


Integral terms appearing in equations (9)-(10) are written analogously. These elements, properly grouped, form the stiffness matrix $\mathbf{K}^{(e)}$ :

$$
\begin{gathered}
K_{(n, m)}^{(e)}=\int_{\Omega^{(e)}}\left[f_{1} \frac{\partial \varphi_{n}}{\partial x} \frac{\partial \varphi_{m}}{\partial x}+f_{3}\left(\frac{\partial \varphi_{n}}{\partial y} \frac{\partial \varphi_{m}}{\partial y}+\frac{\partial \varphi_{n}}{\partial z} \frac{\partial \varphi_{m}}{\partial z}\right)\right] d \Omega^{(e)} \\
K_{(n, m+M)}^{(e)}=\int_{\Omega^{(e)}}\left(f_{2} \frac{\partial \varphi_{n}}{\partial x} \frac{\partial \varphi_{m}}{\partial y}+f_{3} \frac{\partial \varphi_{n}}{\partial y} \frac{\partial \varphi_{m}}{\partial x}\right) d \Omega^{(e)} \\
K_{(n, m+2 M)}^{(e)}=\int_{\Omega^{(e)}}\left(f_{2} \frac{\partial \varphi_{n}}{\partial x} \frac{\partial \varphi_{m}}{\partial z}+f_{3} \frac{\partial \varphi_{n}}{\partial z} \frac{\partial \varphi_{m}}{\partial x}\right) d \Omega^{(e)} \\
K_{(n+M, m)}^{(e)}=\int_{\Omega^{(e)}}\left(f_{2} \frac{\partial \varphi_{n}}{\partial y} \frac{\partial \varphi_{m}}{\partial x}+f_{3} \frac{\partial \varphi_{n}}{\partial x} \frac{\partial \varphi_{m}}{\partial y}\right) d \Omega^{(e)} \\
K_{(n+M, m+M)}^{(e)} \int_{\Omega^{(e)}}\left[f_{1} \frac{\partial \varphi_{n}}{\partial y} \frac{\partial \varphi_{m}}{\partial y}+f_{3}\left(\frac{\partial \varphi_{n}}{\partial x} \frac{\partial \varphi_{m}}{\partial x}+\frac{\partial \varphi_{n}}{\partial z} \frac{\partial \varphi_{m}}{\partial z}\right)\right] d \Omega^{(e)} \\
K_{(n+M, m+2 M)}^{(e)}=\int_{\Omega^{(e)}}\left(f_{2} \frac{\partial \varphi_{n}}{\partial y} \frac{\partial \varphi_{m}}{\partial z}+f_{3} \frac{\partial \varphi_{n}}{\partial z} \frac{\partial \varphi_{m}}{\partial y}\right) d \Omega^{(e)} \\
K_{(n+2 M, m)}^{(e)}=\int_{\Omega^{(e)}}\left(f_{2} \frac{\partial \varphi_{n}}{\partial z} \frac{\partial \varphi_{m}}{\partial x}+f_{3} \frac{\partial \varphi_{n}}{\partial x} \frac{\partial \varphi_{m}}{\partial z}\right) d \Omega^{(e)} \\
K_{(n+2 M, m+M)}^{(e)}=\int_{\Omega^{(e)}}\left(f_{2} \frac{\partial \varphi_{n}}{\partial z} \frac{\partial \varphi_{m}}{\partial y}+f_{3} \frac{\partial \varphi_{n}}{\partial y} \frac{\partial \varphi_{m}}{\partial z}\right) d \Omega^{(e)} \\
\left.K_{(n+2 M, m+2 M)}^{(e)} f_{1} \frac{\partial \varphi_{n}}{\partial z} \frac{\partial \varphi_{m}}{\partial z}+f_{3}\left(\frac{\partial \varphi_{n}}{\partial x} \frac{\partial \varphi_{m}}{\partial x}+\frac{\partial \varphi_{n}}{\partial y} \frac{\partial \varphi_{m}}{\partial y}\right)\right] d \Omega^{(e)}
\end{gathered}
$$

where $n=m=0,1, \ldots, M-1$.

The vector $\mathbf{B}^{(e)}$ contains thermal loads. If the temperature distribution is the only load acting in the analyzed domain, then $p_{x}, p_{y}, p_{z}$ occurring in equations (8)-(10) disappear. The elements of $\mathbf{B}^{(e)}$ are calculated as follows:

$$
\begin{aligned}
B_{n}^{(e)} & =\alpha f_{4} \int_{\Omega^{(e)}} \frac{\partial \varphi_{n}}{\partial x} \Delta T_{n} d \Omega^{(e)} \\
B_{n+M}^{(e)} & =\alpha f_{4} \int_{\Omega^{(e)}} \frac{\partial \varphi_{n}}{\partial y} \Delta T_{n} d \Omega^{(e)} \\
B_{n+2 M}^{(e)} & =\alpha f_{4} \int_{\Omega^{(e)}} \frac{\partial \varphi_{n}}{\partial z} \Delta T_{n} d \Omega^{(e)}
\end{aligned}
$$


After calculating the integrals (16)-(17) and inserting them into the matrix $\mathbf{K}^{(e)}$ and the vector $\mathbf{B}^{(e)}$, a local FEM system of equations is obtained.

The discrete model of the thermoelasticity problem currently consists of $N$ local stiffness matrices $\mathbf{K}^{(e)}$ and local right side vectors $\mathbf{B}^{(e)}$. In order to build a global model, one needs to sum up the local matrices:

$$
\begin{aligned}
\mathbf{K} & =\sum_{i=0}^{N-1} \mathbf{K}^{(i)} \\
\mathbf{B} & =\sum_{i=0}^{N-1} \mathbf{B}^{(i)}
\end{aligned}
$$

Aggregation is performed based on node numbers in the finite element mesh. The size of the global matrix depends on the number of nodes and the number of unknowns on each node. In this task, the unknowns are the components of the displacement vector $u_{x}, u_{y}, u_{z}$, i.e. there are $3 L$ unknowns at the $L$ nodes in the entire mesh. The global system of FEM equations is presented below:

$$
\mathbf{K} \cdot \mathbf{u}=\mathbf{B}
$$

After solving the system of equations (20), the displacement vector $\mathbf{u}$ is obtained. To determine the strains in finite elements the following geometric relationships are used:

$$
\begin{array}{ll}
\varepsilon_{x}=\frac{\partial u_{x}}{\partial x}, & \gamma_{x y}=\gamma_{y x}=\frac{\partial u_{x}}{\partial y}+\frac{\partial u_{y}}{\partial x} \\
\varepsilon_{y}=\frac{\partial u_{y}}{\partial y}, & \gamma_{y z}=\gamma_{z y}=\frac{\partial u_{y}}{\partial z}+\frac{\partial u_{z}}{\partial y} \\
\varepsilon_{z}=\frac{\partial u_{z}}{\partial z}, & \gamma_{x z}=\gamma_{z x}=\frac{\partial u_{x}}{\partial z}+\frac{\partial u_{z}}{\partial x}
\end{array}
$$

The values $\varepsilon_{x}, \varepsilon_{y}, \varepsilon_{z}$ obtained on their basis relationship (21) are the normal strains while $\gamma_{x y}, \gamma_{y x}, \gamma_{y z}, \gamma_{z y}, \gamma_{x z}, \gamma_{z x}$ are the shear strains. The values of elastic strains $\varepsilon_{s x}, \varepsilon_{s y}, \varepsilon_{s z}$ are obtained by the following way:

$$
\begin{gathered}
\varepsilon_{s x}=\varepsilon_{x}-\varepsilon_{T}=\frac{\partial u_{x}}{\partial x}-\alpha \Delta T \\
\varepsilon_{s y}=\varepsilon_{y}-\varepsilon_{T}=\frac{\partial u_{y}}{\partial y}-\alpha \Delta T \\
\varepsilon_{s z}=\varepsilon_{z}-\varepsilon_{T}=\frac{\partial u_{z}}{\partial z}-\alpha \Delta T
\end{gathered}
$$


Normal stresses $\sigma_{x}, \sigma_{y}, \sigma_{z}$ in the elements are as follows:

$$
\begin{aligned}
& \sigma_{x}=\frac{E}{1+v}\left[\varepsilon_{s x}+\frac{v}{1-2 v}\left(\varepsilon_{s x}+\varepsilon_{s y}+\varepsilon_{s z}\right)\right] \\
& \sigma_{y}=\frac{E}{1+v}\left[\varepsilon_{s y}+\frac{v}{1-2 v}\left(\varepsilon_{s x}+\varepsilon_{s y}+\varepsilon_{s z}\right)\right] \\
& \sigma_{z}=\frac{E}{1+v}\left[\varepsilon_{s z}+\frac{v}{1-2 v}\left(\varepsilon_{s x}+\varepsilon_{s y}+\varepsilon_{s z}\right)\right]
\end{aligned}
$$

After creating the geometry of the object and spatial discretization, which makes it possible to obtain a set of finite elements, the calculations are carried out in three stages:

- determination of the nodal displacements,

- calculation of the strains in the finite elements,

- calculation of the stresses in the finite elements.

\section{Example of calculations}

The 3D-region shown in Figure 3 was prepared to obtain the numerical solution of the problem of thermoelasticity.

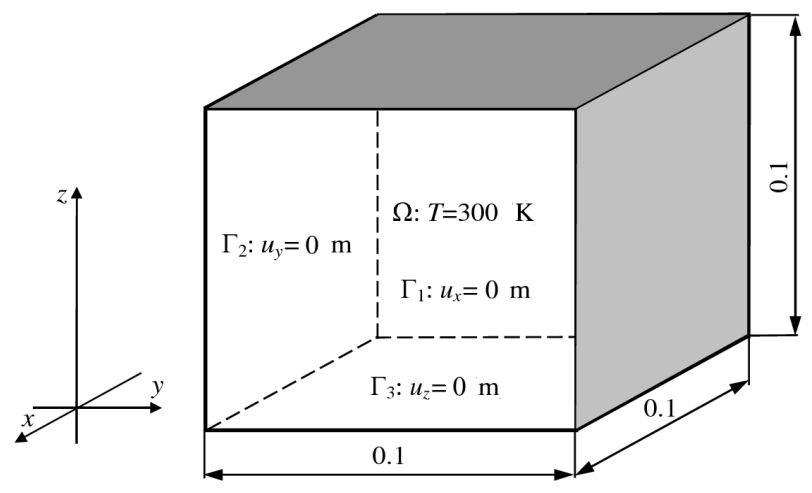

Fig. 3. A heat-loaded cubic region used in the calculations

The material properties of steel used for calculations are presented in Table 1.

Table 1. Material properties used in calculations

\begin{tabular}{|l|c|}
\hline \multicolumn{1}{|c|}{ Material parameter } & Value \\
\hline Young's modulus $E\left[\mathrm{~N} / \mathrm{m}^{2}\right]$ & $2 \cdot 10^{11}$ \\
\hline Poisson number $v[-]$ & 0.32 \\
\hline Coefficient of thermal expansion $\alpha\left[\mathrm{K}^{-1}\right]$ & $1.2 \cdot 10^{-5}$ \\
\hline
\end{tabular}


The heated cubic element is able to increase its volume without any obstacles, which means that the supports do not generate stress. Thermal deformations arise only in the cubic element, the value of which can easily be calculated, while elastic deformations are equal to zero. Increasing the temperature by $300 \mathrm{~K}$ causes thermal deformations in each finite element equal to $\varepsilon_{T}=\alpha \Delta T=1.2 \cdot 10^{-5} \cdot 300=3.6 \cdot 10^{-3}$, while the corresponding displacements in the each direction are the same and equal to $\varepsilon_{T} L=3.6 \cdot 10^{-3} \cdot 0.1=3.6 \cdot 10^{-4} \mathrm{~m}$ on the free walls.

The cube was divided into finite elements. Spatial discretization was carried out using the GMSH mesh generator. The average dimension of the element was assumed equal, resulting in a mesh of 5126 tetrahedrons.

The results of numerical calculations showed full compliance with the theory. Components of the displacement vector measured on non-attached walls with a direction perpendicular to the attached walls reach values equal to $3.6 \cdot 10^{-4} \mathrm{~m}$ with negligible small errors.

Figures $4 \mathrm{a}-\mathrm{c}$ present the values of the components of displacement vectors calculated in the nodes using the second order of approximation.
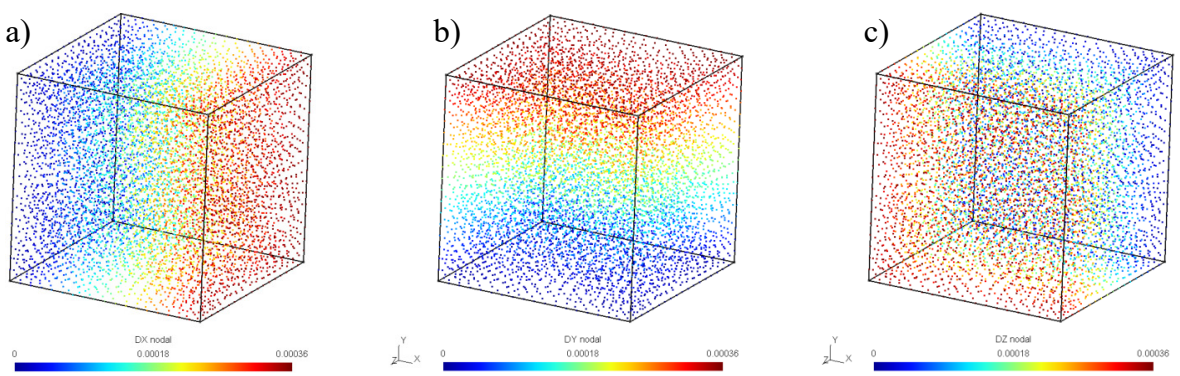

Fig. 4. Values of displacement components in directions: a) $x$, b) $y$, c) $z$

Figures 5a-b present displacement fields obtained as a result of calculations carried out using the first and second order of approximation. The values obtained are almost identical.

a)

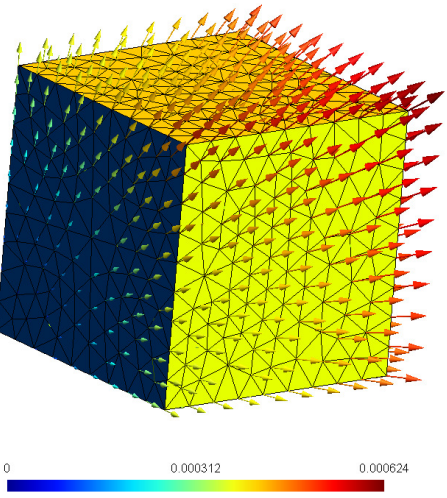

b)

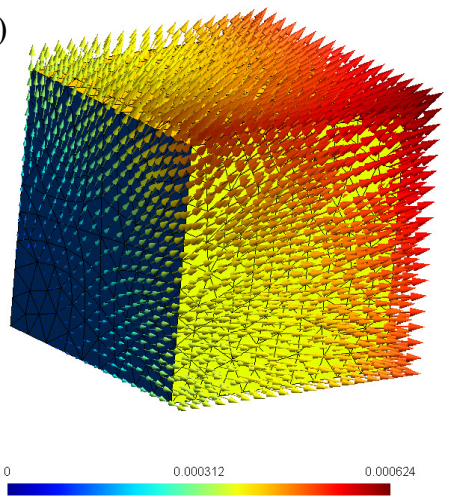

Fig. 5. Displacement vectors in grid nodes obtained for approximation: a) first, b) second orders 


\section{Conclusions}

The obtained results confirm the correctness of the solver based on the continuous Galerkin formulation, which is an alternative to the discontinuous version of the method [9] used to solve the problem of thermoelasticity. The created original solver allows for numerical computations using complex three-dimensional meshes. Calculations are time efficient even for a large number of nodes. Modifications are also easier to implement than in the case of expensive commercial packages. The future work is focused on the comparison of the obtained results to the results of a/the discontinuous approach.

\section{References}

[1] Nowacki, W. (1965). Dynamiczne zagadnienia termosprężystości. Mechanika Teoretyczna i Stosowana, 3(3), 3-49.

[2] Qin, Q.H. (2000). The Trefftz Finite and Boundary Element Methods. Southampton: WIT Press.

[3] Kleiber, M. (1995). Komputerowe metody mechaniki ciat statych. Warszawa: PWN.

[4] Kincaid, D., \& Cheney, W. (2006). Analiza numeryczna. Warszawa: WNT.

[5] Majchrzak, E., Mochnacki, B. (2004). Numerical methods. Theory, numerical aspects, algorithms. Gliwice: Publication of the Silesian University of Technology.

[6] Cichoń, C. (2005). Metody obliczeniowe. Wybrane zagadnienia. Kielce: Wydawnictwo Politechniki Świętokrzyskiej.

[7] Zienkiewicz, O.C. (1977). The Finite Element Method. London: Mc Graw-Hill.

[8] Bathe, K.J. (1982). Finite Element Procedures in Engineering Analysis. Prentice-Hall.

[9] Węgrzyn-Skrzypczak, E. (2019). Discontinuous Galerkin method for the three-dimensional problem of thermoelasticity. Journal of Applied Mathematics and Computational Mechanics, 18(4), 115-126. 